\title{
Fuzzy recurrence plots
}

\author{
Tuan Pham
}

Journal Article

Tweet

N.B.: When citing this work, cite the original article.

Original Publication:

Tuan Pham , Fuzzy recurrence plots, Europhysics letters, 2016. 116(), pp.p1-p5. http://dx.doi.org/10.1209/0295-5075/116/50008

Copyright: European Physical Society

http://www.eps.org/

Postprint available at: Linköping University Electronic Press

http://urn.kb.se/resolve?urn=urn:nbn:se:liu:diva-134393

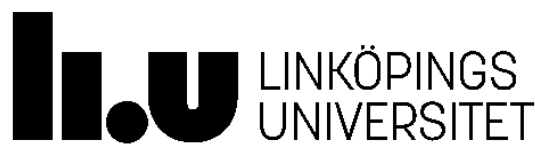




\title{
Fuzzy Recurrence Plots
}

\author{
T.D. РHAM
}

Department of Biomedical Engineering

Linkoping University

58183 Linkoping, Sweden

PACS 05.45.Tp - Time series analysis

PACS 07.05.Mh - Neural networks, fuzzy logic, artificial intelligence

PACS 07.05.Rm - Data presentation and visualization: algorithms and implementation

\begin{abstract}
Recurrence plots display binary texture of time series from dynamical systems with single dots and line structures. Using fuzzy recurrence plots, recurrences of the phase-space states can be visualized as grayscale texture, which is more informative for pattern analysis. The proposed method replaces the crucial similarity threshold required by symmetrical recurrence plots with the number of cluster centers, where the estimate of the latter parameter is less critical than the estimate of the former.
\end{abstract}

Introduction. - The notion of recurrence plots was introduced as a graphical tool for studying dynamical systems in chaos theory [1], and examined in detail ten years later [2]. A recurrence plot $(\mathrm{RP})$ can be easily constructed to display useful information to aid the analysis of a complex system, and also be further analyzed with various measures of recurrence quantification [3]. The applications of RPs have been increasingly found in many areas of research [4], where typically most recent reports include cell biology [5], mild cognitive impairment in type 2 diabetes [6], human brain-age prediction on magnetic resonance imaging [7], musical structure [8], combustion noise [9], and carbon steel corrosion [10].

Given the popularity of RPs, a particular problem with the graphic representation of RPs is the appropriate selection of the similarity thresholds that are sensitive for the visualization of the recurrence patterns of dynamical systems [11]. Here, the concept of fuzzy relations is introduced to measure the similarity of two phase-space states of a trajectory in time-series data on the basis of a continuum of grades of membership ranging between zero and one. Not only the use of such a relation can alleviate the problem of the threshold selection, it also enhances the visualization of the system dynamics with more detail of texture that is hardly discovered in experimental data.

Recurrence plots. - Let $X=\{x\}$ be a set of phasespace states, in which $x_{i}$ is the $i$-th state of a dynamical system in $m$-dimensional space. An $\mathrm{RP}$ is an $N \times N$ matrix in which an element $(i, j), i=1, \ldots, N, j=1, \ldots, N$, is represented with a black dot if $x_{i}$ and $x_{j}$ are considered to be closed to each other [1]. State $x_{j}$ is close to state $x_{i}$ if $x_{j}$ is within the radius $r_{i}$, which should contain an appropriate number of other states, where $x_{i}$ is the center of the radius [1]. This condition can result in an asymmetrical RP, because by definition, $r_{i}$ and $r_{j}$ may not be the same.

In order to construct a symmetrical RP, a threshold, denoted as $\epsilon$, is used to define the closeness or similarity of a state pair $\left(x_{i}, x_{j}\right)$ as follows [3]:

$$
R(i, j)=H\left(\epsilon-\left\|x_{i}-x_{j}\right\|\right)
$$

where $R(i, j)$ is an element $(i, j)$ of the recurrence matrix $R$, and $H(\cdot)$ is the Heaviside step function or the unit step function that yields either 0 or 1 if $\left(\epsilon-\left\|x_{i}-x_{j}\right\|\right)<0$ or otherwise, respectively.

Fuzzy recurrence plots. - Let $X=\{x\}$, and $V=$ $\{v\}$ be the sets of phase-space states and fuzzy clusters of the states, respectively. A fuzzy (binary) relation $R$ from $X$ to $V$ is a fuzzy subset of $X \times V$ characterized by a fuzzy membership (characteristic) function $\mu \in[0,1]$. This (fuzzy) grade of membership expresses the similarity or strength of relation of each pair $(x, v)$ in $R$ that has the following properties [12].

1. Reflexivity:

$$
\mu(x, x)=1, \forall x \in X .
$$

2. Symmetry: 


$$
\mu(x, v)=\mu(v, x), \forall x \in X, \forall v \in V .
$$

\section{Transitivity:}

$\mu(x, z)=\vee_{v}[\mu(x, v) \wedge \mu(v, z)], \forall x \in X, \forall z \in Z$, which is called the max-min composition, where the symbols $\checkmark$ and $\wedge$ stand for max and min, respectively.

The fuzzy clusters of the phase-space states can be obtained using the fuzzy $c$-means (FCM) algorithm $[13,14]$ to determine the closeness or similarity between the states and their fuzzy cluster centers in order to subsequently infer the similarity between the pairs of the states using the max-min composition (transitivity) of a fuzzy relation.

Let $\left\{x_{1}, x_{2}, \ldots, x_{N}\right\}$ be a set of phase-space states, the FCM algorithm works by minimizing the following fuzzy objective function [13]:

$$
J(U, Z)=\sum_{i=1}^{N} \sum_{j=1}^{c}\left(\mu_{i j}\right)^{m}\left[d\left(x_{i}, z_{j}\right)\right]^{2},
$$

where $c$ is the number of clusters, $1<c<N, m \in[1, \infty)$ is the fuzzy weighting exponent ( $m=2$ in this study), $U=\left[\mu_{i j}\right], i=1, \ldots, N, j=1, \ldots, c$, is the matrix of the fuzzy $c$-partition, $Z=\left(z_{1}, z_{2}, \ldots, z_{c}\right)$ is the vector of cluster centers, $z_{j}$ is the center of cluster $j$, and $d\left(x_{i}, z_{j}\right)$ is any inner-product induced norm metric (Euclidean metric was used in this study).

The above fuzzy objective function is subject to

$$
\sum_{j=1}^{c} \mu_{i j}=1, i=1, \ldots, N
$$

where $\mu_{i j} \in[0,1], i=1, \ldots, N, j=1, \ldots, c$.

In order to optimally determine $U$ and $Z$, a numerical solution to the minimization of the objective function $J(U, Z)$ is by an iterative process of updating $U$ and $Z$ until some convergence is reached. The fuzzy membership grades and cluster centers of the FCM are updated as [22]

$$
\begin{gathered}
\mu_{i j}=\frac{1}{\sum_{j=1}^{c}\left[\frac{d\left(x_{i}, z_{k}\right)}{d\left(x_{i}, z_{j}\right)}\right]^{2 /(m-1)}}, 1 \leq k \leq c ; \\
z_{j}=\frac{\sum_{i=1}^{N}\left(\mu_{i j}\right)^{m} x_{i}}{\sum_{i=1}^{N}\left(\mu_{i j}\right)^{m}}, \forall j .
\end{gathered}
$$

The updating process is stopped if $\left\|U^{t}-U^{t+1}\right\| \leq \epsilon$, where $t$ is the $t$-th time step, and $\epsilon$ is a small positive real number that indicates the level of accuracy $(\epsilon=0.00001$ in this study).

Thus, a fuzzy recurrence plot (FRP), which is symmetrical and considered as a fuzzy relation matrix of the phasespace states, can be visualized as a grayscale image whose intensity values are represented with the fuzzy membership grades of similarity of the state pairs. To be consistent with the visual display of an RP where a black dot is located at a state pair that is considered to be close to each other, the image intensities in the range $[0,1]$ of an
FRP are represented with the complements of the fuzzy membership grades $(1-\mu(x, z))$, which give a black pixel if $x=z$.

Examples. - Three examples are given to illustrate advantages of FRP over RP with respect to visual effects and parameter specification of dynamical systems. The first example is the time series of the $x$-component (angular velocity) of the Lorenz system [15], the second example is the time series of electromyography (EMG) signals of human aggressively physical action of elbowing [16], and the third example is the pseudo periodic synthetic time series [16].

The data types were selected with a purpose to compare the graphical displays of texture generated with RP and FRP from the time series of a chaotic behavior (Lorentz system), dynamic pattern of a human action (EMG signals), and combined deterministic and random phenomenon (pseudo periodic synthetic time series). In particular, the study of primitives of human motions for automated recognition of certain types of human action patterns using physiological (EMG) signals and images is an important area of research with wide-ranging applications in the diagnosis of physical or mental disorder, prosthesis and rehabilitation devices, human-machine interactions, security, surveillance, assistive technologies for improving quality of life, and so on [17-20].

The lengths of the Lorenz system, elbowing, and pseudo periodic synthetic time series are 4,000, 100,000, and 9,772 instances, respectively. Figure 1 shows the plots of these three time series. The pseudo periodic synthetic time series were produced using the equation $y=$ $\sum_{i=3}^{7}\left(1 / 2^{i}\right) \sin \left[2 \pi\left(2^{2+i}+\operatorname{rand}\left(2^{i}\right)\right) t\right], 0 \leq t \leq 1$, where the output of $\operatorname{rand}(x)$ is a random integer between 0 and $x$ [16]. This synthetic data was designed for testing indexing schemes in time series databases, and are highly periodic but do not have any repeats [16]. Because the calculation of the recurrences for the pseudo periodic synthetic time series of 100,000 instances requested more than 200 GB that exceeds the maximum array size of a personal computer Probook 6570b with Core i7, the creation of arrays greater than this limit may take a long time and cause MATLAB to become unresponsive. Therefore, the first 10,000 instances of the synthetic time series were used in this study.

Figures 2, 3, and 4 show the RPs and FRPs of the Lorenz system, EMG signals of elbowing, and pseudo periodic synthetic time series, respectively, with embedding dimension $=1$, time delay $=1$, and various values of the threshold $\epsilon$ for RPs and number of clusters for FRPs. The threshold $\epsilon$ was suggested to take values not being greater than $10 \%$ of the mean of the data [3], hence $10 \%, 5 \%$, and $1 \%$ were selected in this study. For the FRPs, the numbers of clusters to be specified in the FCM were arbitrarily selected as 2, 3, and 5. The number of clusters in FRPs are inversely proportional to the threshold magnitude of RPs, i.e., smaller number of clusters allow more states to 
be close to each other, making the plots denser with darker pixels.

In all three examples, the FRPs with the content of the grayscale texture, particularly as shown in Figures 2 and 4 , are more informative than the binary RPs, leading to better visualization of the characteristics of dynamical systems in phase space. The RPs of the $x$-component of the Lorenz system can be obtained with more textural information when the dynamical parameters (embedding dimension, time delay, threshold, and sampling time) are carefully selected [3]; while these tasks can be relaxed with the use of the FRP method. The RPs of the pseudo periodic synthetic time series as shown in Figure 4 (a)-(c) lack clear visual displays of diagonal lines and checkerboard structures that can be observed in the FRPs shown in Figure 4 (d)-(f). Both RPs and FRPs show similar recurrence patterns for the EMG signals of elbowing. In general, grayscale patterns of the recurrences of phasespace states convey subtle behaviors of dynamic systems, which can be more effective for recurrence quantification analysis.

Conclusion. - Measure of closeness of the phasespace states using the concepts of fuzzy relations where the fuzzy grades of membership can be obtained by the FCM appears to be a natural way of quantification with respect to recurrences. FRPs are richer in texture giving better visualization and easier in parameter specification than RPs. In fact, optimal estimates of the number of clusters for the FCM algorithm can be obtained using fuzzy cluster validity $[13,21,22]$. The proposed FRP method is promising to be a useful tool for texture analysis and classification of time series, which are pervasive in many applications.

The Matlab code for implementing the fuzzy recurrence plots is available at the author's personal homepage: https://sites.google.com/site/professortuanpham/codes.

\section{REFERENCES}

[1] ECKMANN J.P., KAMPHORST S.O. and RUELLE D., Europhysics Letters, 4 (1987) 973.
[2] CASDAGLI M.C., Physica D, 108 (1997) 12.

[3] MARWAN N., ROMANO M.C., THIEL M. and KURTHS J., Physics Reports, 438 (2007) 237.

[4] MARWAN N., SCHINKEL S. and KURTHS J., EPL, 101 (2013) 20007.

[5] HIRATA Y., ODA A., OHTA K. and AIHARA K., Scientific Reports, 6 (2016) 34982.

[6] CUI D., WANG J., WANG L., YIN S., BIAN Z. and GU G., Neurocomputing, 203 (2016) 102.

[7] PHAM T.D., ABE T., OKA R. and CHEN Y.F., Entropy, 17 (2015) 8130.

[8] FUKINO M., HIRATA Y. and AIHARA K., Chaos, 26 (2016) 023116.

[9] KABIRAJ L., SAURABH A., NAWROTH H. and PASCHEREIT C.O., AIAA Journal, 53 (2015) 1199.

[10] HOU Y., ALDRICH C., LEPKOVA K., MACHUCA L.L. and KINSELLA B., Corrosion Science, 112 (2016) 63.

[11] BIEM GRABEN P., SELLERS K.K., FROHLICH F. and HUTT A., EPL, 114 (2016) 38003.

[12] ZADEH L.A., Information Sciences, 3 (1971) 177.

[13] BEZDEK J.C., Pattern Recognition with Fuzzy Objective Function Algorithms (Plenum Press) 1981.

[14] BEZDEK J.C., EHRLICH R. and FULL W., Computers \& Geosciences, 10 (1984) 191.

[15] LORENZ E.N., Journal of the Atmospheric Sciences, 20 (1963) 130.

[16] LICHMAN M., UCI Machine Learning Repository (University of California, School of Information and Computer Science, Irvine, CA, USA) 2013, http://archive.ics.uci.edu/ml (Last accessed 24 October 2016).

[17] CHU J.U., MOON I. and MUN M.S., IEEE Transactions on Biomedical Engineering, 53 (2006) 2232.

[18] CHOWDHURY R.H., et al., Sensors, 13 (2013) 12431.

[19] DEL VECCHIO D., MURRAY R.M. and PERONA P., Proceedings of 2003 European Control Conference, 2003, pp. $445-450$.

[20] IJJINA E.P. and MOHAN C.K., Pattern Recognition Letters, 83 (2016) 268.

[21] WANG W. and ZHANG Y., Fuzzy Sets and Systems, 158 (2007) 2095.

[22] BEZDEK J.C., MOSHTAGHI M., RUNKER T. and LECKIE C., The generalized $C$ index for internal fuzzy cluster validity, to be published in IEEE Transactions on Fuzzy Systems (2016). DOI: 10.1109/TFUZZ.2016.2540063. 

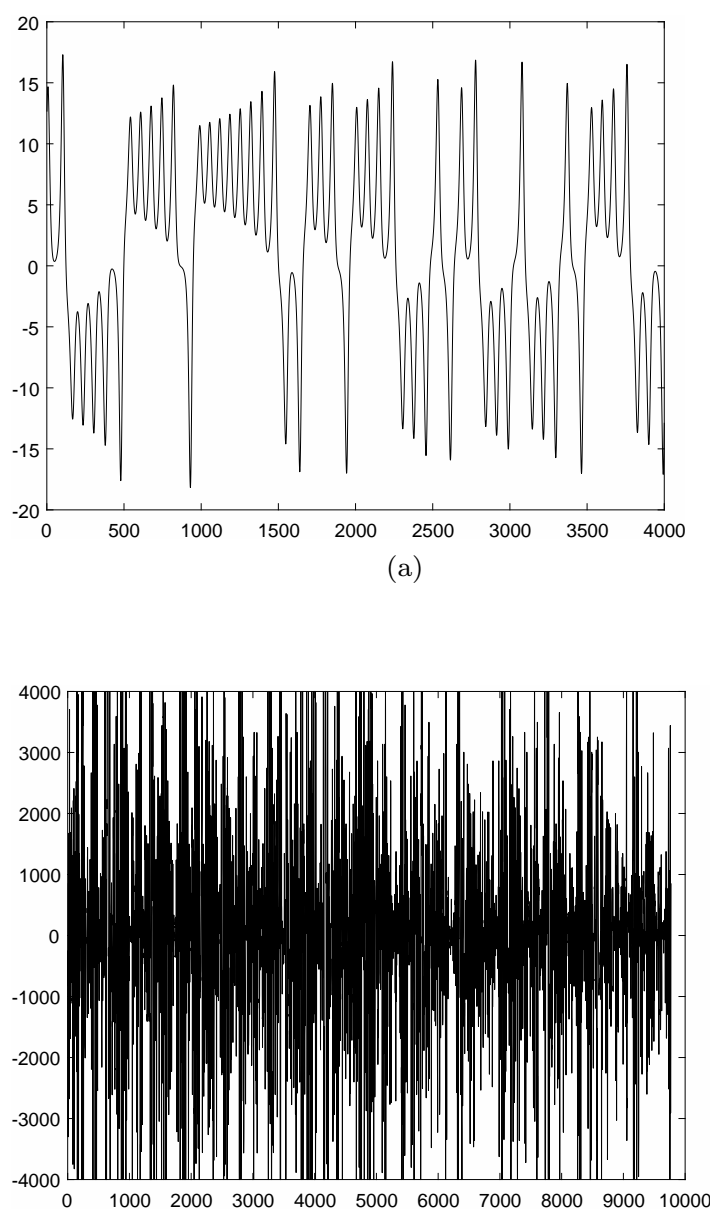

(b)

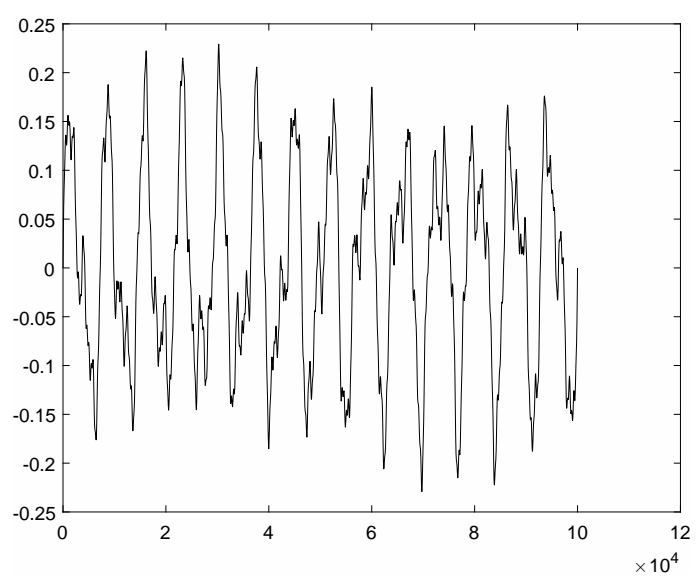

(c)

Fig. 1: (a) The $x$-component of the Lorenz system (b) EMG signals of human elbowing action, and (c) pseudo periodic synthetic time series. 


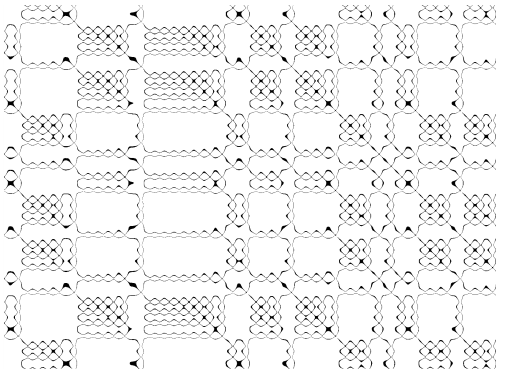

(a)

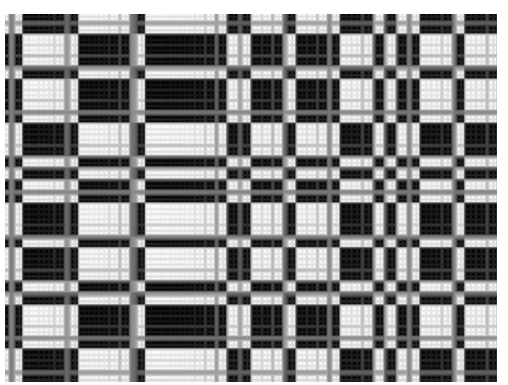

(d)

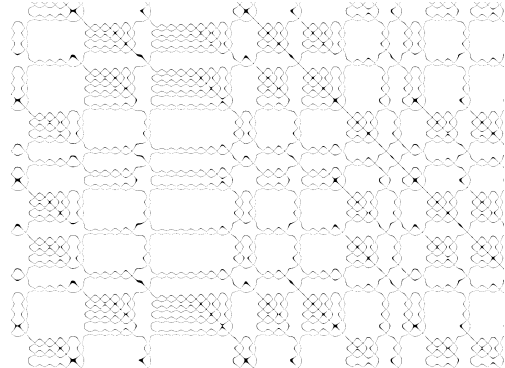

(b)

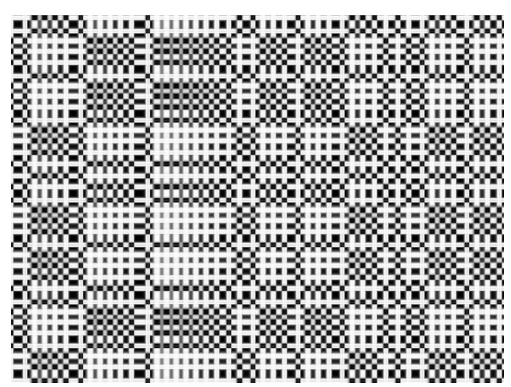

(e) (c)

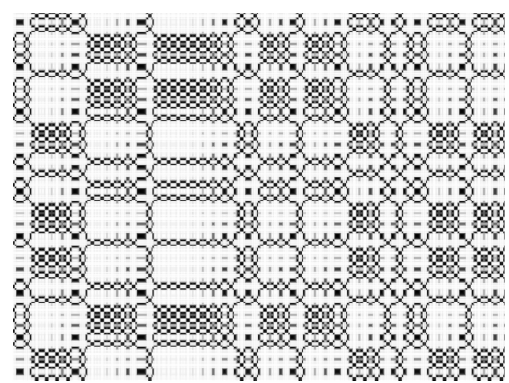

(f)

Fig. 2: Recurrences of phase-space states of the $x$-component of the Lorenz system, with embedding dimension $=$ 1, and time delay =1: (a), (b), and (c) are recurrence plots with threshold $\epsilon=10 \%$, $5 \%$, and $1 \%$ of the mean, respectively; and (d), (e), and (f) are fuzzy recurrence plots with the number of clusters $=2$, 3 , and 5 , respectively.

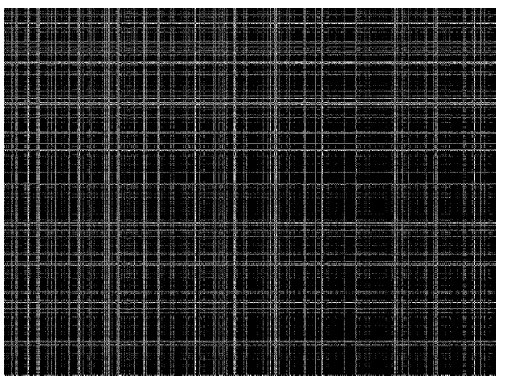

(a)

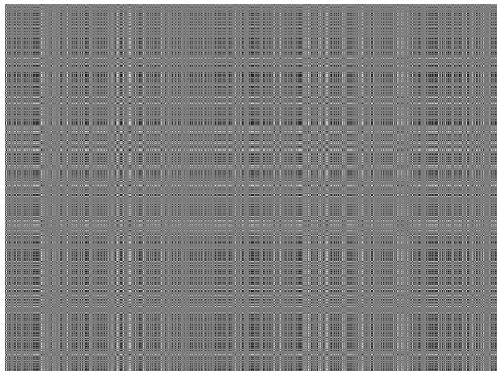

(d)

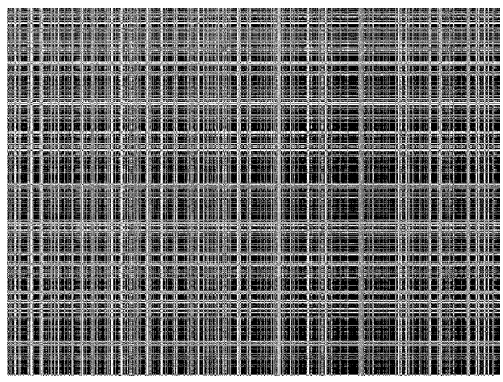

(b)

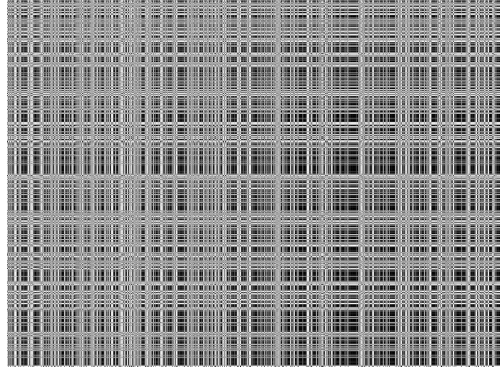

(e)

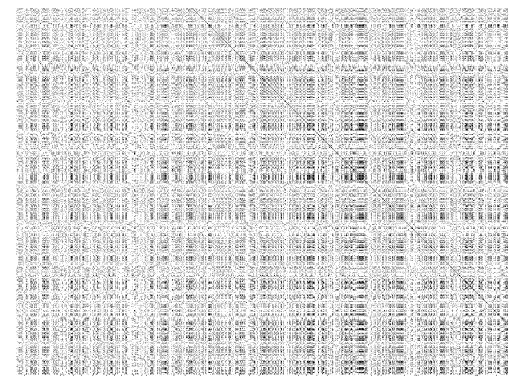

(c)

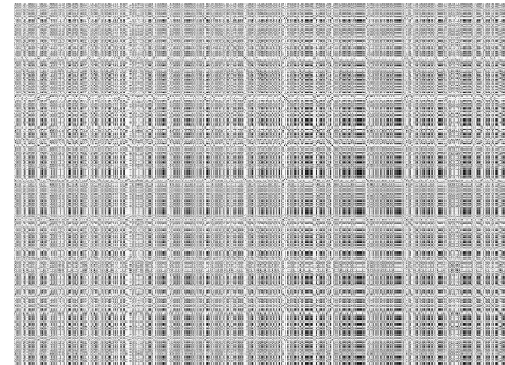

(f)

Fig. 3: Recurrences of phase-space states of an elbowing action, with embedding dimension $=1$, and time delay $=1$ : (a), (b), and (c) are recurrence plots with threshold $\epsilon=10 \%, 5 \%$, and $1 \%$ of the mean, respectively; and (d), (e), and (f) are fuzzy recurrence plots with the number of clusters $=2,3$, and 5 , respectively. 


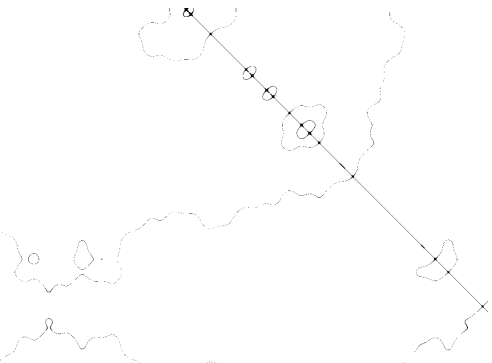

(a)

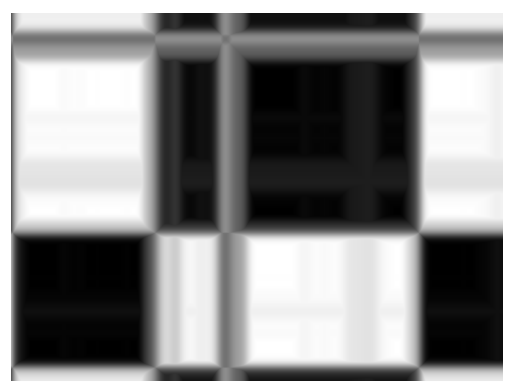

(d)

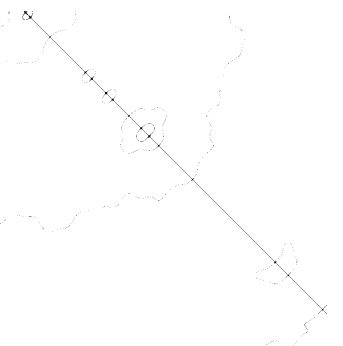

(b)

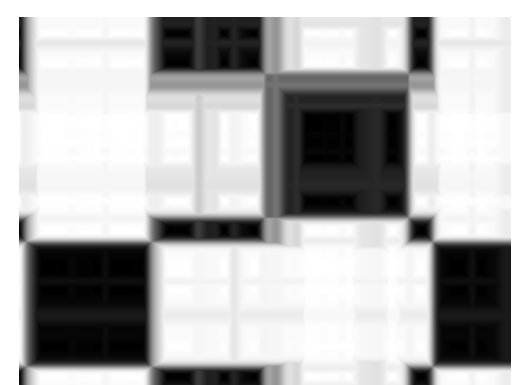

(e) (c)

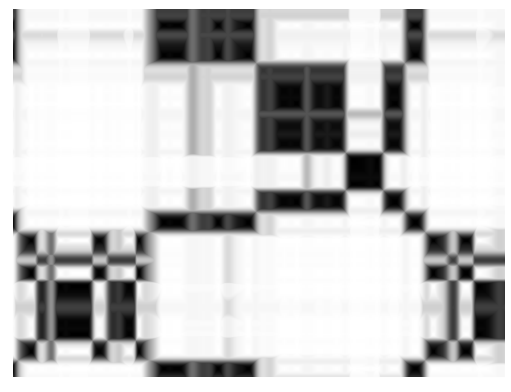

(f)

Fig. 4: Recurrences of phase-space states of pseudo periodic synthetic time series, with embedding dimension $=1$, and time delay $=1:(\mathrm{a}),(\mathrm{b})$, and (c) are recurrence plots with threshold $\epsilon=10 \%, 5 \%$, and $1 \%$ of the mean, respectively; and (d), (e), and (f) are fuzzy recurrence plots with the number of clusters $=2,3$, and 5 , respectively. 